\title{
REMARKS ON THE MAXIMAL DILATATION OF THE BEURLING-AHLFORS EXTENSION
}

\author{
MATTI LEHTINEN
}

\section{Introduction}

An increasing continuous function $h$ defined on an interval $I$, which can be the entire real line $R^{1}$, is $\varrho$-quasisymmetric if

$$
\varrho^{-1} \leqq(h(x+t)-h(x)) /(h(x)-h(x-t)) \leqq \varrho
$$

for all $x$ and all $t>0$ such that $[x-t, x+t] \subset I$. The infimum $\varrho(h)$ of all $\varrho$ such that (1) holds is the coefficient of quasisymmetry of $h$.

A well-known and widely applied result due to Beurling and Ahlfors [1] states that the map $f_{h, r}$ defined by

where

$$
2 f_{h, r}(z)=\alpha(z)+\beta(z)+\operatorname{ir}(\alpha(z)-\beta(z)),
$$

$$
\alpha(z)=\int_{0}^{1} h(x+y t) d t, \quad \beta(z)=\int_{-1}^{0} h(x-y t) d t, \quad z=x+i y, \quad r>0,
$$

is a quasiconformal extension of $h$ to a domain $A$ in the upper half-plane $H$. If $I=R^{1}$, then $A=H$, and if $I$ is a finite interval, $A$ is a right isosceles triangle with base $I$. We call $f_{h, r}$ a Beurling-Ahlfors extension of $h$.

Beurling and Ahlfors proved the existence of an $r$, depending on $\varrho$ only, such that the maximal dilatation $K\left(f_{h, r}\right)$ of $f_{h, r}$ is at most $\varrho^{2}$ as soon as $\varrho(h) \leqq \varrho$. For $\varrho \leqq 1.9, \varrho^{2}$ has been replaced by $\varrho^{3 / 2}[3]$. Linear upper bounds $K\left(f_{h, 1}\right) \leqq 8 \varrho, K\left(f_{h, 1}\right) \leqq$ $4.2 \varrho$ and $K\left(f_{h, 1}\right) \leqq 2 \varrho$ have been computed by T. Reed [6], Li Zhong [5] and the author [4], respectively. In the present paper, the implications of (1) on $K\left(f_{h, r}\right)$ will be examined in greater detail. The main result is that for each $\varrho$ there is an $r$ such that

$$
K\left(f_{h, r}\right) \leqq 2 \varrho-1 .
$$

In the other direction, we shall give an example of a piecewise linear $h$ such that

for every $r$.

$$
K\left(f_{h, r}\right)>3 \varrho / 2
$$




\section{A lemma of Beurling and Ahlfors}

The upper estimations of $K\left(f_{h, r}\right)$ make use of the following lemma of Beurling and Ahlfors [1, p. 137]:

Lemma 1. Let $h$ be $\varrho$-quasisymmetric on $[0,1], h(0)=0, h(1)=1$. Set $\mu=$ $\varrho /(\varrho+1), \lambda=1 /(\varrho+1)$. Then

$$
\lambda \leqq \int_{0}^{1} h(x) d x \leqq \mu .
$$

We reprove the lemma with a method indicating a function which yields equality on the right-hand side of (3). Set $P(0)=0, P(1)=1$ and

$$
P\left((2 k+1) / 2^{n}\right)=\lambda P\left(k / 2^{n-1}\right)+\mu P\left((k+1) / 2^{n-1}\right),
$$

$k=0,1, \ldots, 2^{n-1}-1 ; n=1,2, \ldots$, and extend the definition of $P$ to numbers $x \in(0,1)$ with a non-terminating binary representation by continuity. ( $P$ is the function introduced by R. Salem [7] and studied in relation to quasisymmetric functions by K. Goldberg [2].) Clearly

$$
h(x) \leqq P(x)
$$

for all $x \in[0,1]$ with a finite binary representation, and by continuity on the whole interval. By (4) one computes using induction

which implies

$$
\sum_{k=1}^{2^{n}} P\left(k / 2^{n}\right)=2^{n} \mu+\lambda,
$$

$$
\int_{0}^{1} P(x) d x=\mu .
$$

The right-hand side of (3) now follows from (6) and (5), and the left-hand side from the replacement of $h(x)$ by $1-h(1-x)$.

If the domain of $h$ is $[a, b]$ instead of $[0,1]$, an appropriate linear transformation gives (3) the form

$$
\mu h(a)+\lambda h(b) \leqq(b-a)^{-1} \int_{a}^{b} h(x) d x \leqq \lambda h(a)+\mu h(b) .
$$

Also, (4) and (6) imply

$$
\int_{k / 2^{n}}^{(k+1) / 2^{n}} P(x) d x=\lambda P\left(k / 2^{n}\right)+\mu P\left((k+1) / 2^{n}\right) .
$$

The function $P$ is not quasisymmetric, since e.g.

$$
\left(P\left(1 / 2+1 / 2^{k}\right)-P(1 / 2)\right) /\left(P(1 / 2)-P\left(1 / 2-1 / 2^{k}\right)\right)=\varrho^{k-2} .
$$

This fact together with (5) and (6) indicates that the bounds in (3) are not the best possible ones. A step towards improving (3) is

Lemma 2. Let $[a, b] \subset[0,1]$ and set $c=(a+b) / 2$. If

$$
2 P(c) \leqq P(a)+P(b),
$$


and $h$ is as in Lemma 1 , then

$$
\int_{a}^{b} h(x) d x \leqq(\lambda(b-a) / 2)((\varrho+3) P(c)+(\varrho-1) P(b)) .
$$

Proof. Set

An integration of

$$
X=\int_{a}^{c} h(x) d x, \quad Y=\int_{c}^{b} h(x) d x
$$

over $[c, b]$ yields

$$
h(c+x)-h(c) \leqq \varrho(h(c)-h(c-x))
$$

$$
X+Y \leqq(\varrho+1) h(c)(b-a) / 2 \leqq(\varrho+1) P(c)(b-a) / 2=Z_{0} .
$$

On the other hand, by (7) and (5),

$$
\begin{aligned}
& X \leqq(b-a)(\lambda P(a)+\mu P(c)) / 2=X_{0}, \\
& Y \leqq(b-a)(\lambda P(c)+\mu P(b)) / 2=Y_{0} .
\end{aligned}
$$

Since $\varrho \geqq 1$, the sum $X+Y$ is bounded above by

$$
Y_{0}+\left(Z_{0}-Y_{0}\right) / \varrho
$$

$$
\varrho X_{0}+Y_{0} \geqq Z_{0} \text {. }
$$

But (9) and (12) are equivalent, and the right-hand side of (10) equals (11).

The same argument can be carried over to an arbitrary interval to prove

Lemma 3. Let $h$ be $\varrho$-quasisymmetric on $[a, b]$ and $2 h(c) \leqq h(a)+h(b)$, $c=(a+b) / 2$. Then

$$
\int_{a}^{b} h(x) d x \leqq(\lambda(b-a) / 2)((\varrho+3) h(c)+(\varrho-1) h(b)) .
$$

We next apply Lemma 2 to certain subintervals of $[0,1]$ to obtain the following improvement of Lemma 1:

Lemma 4. If $h$ is $\varrho$-quasisymmetric on $[0,1], h(0)=0, h(1)=1$, then

$$
p \leqq \int_{0}^{1} h(x) d x \leqq q,
$$

where $q=\mu-(5 / 24)\left(\varrho^{2}-\varrho\right) /(\varrho+1)^{4}, \quad p=1-q$.

Proof. By (4), $P\left(1 / 2^{n}\right)=\mu^{n}, P\left(3 / 2^{n}\right)=\mu^{n-1}(1+\lambda), P\left(5 / 2^{n}\right)=\mu^{n-2}(1+\lambda \mu)$, and

$$
P\left(5 / 2^{n}\right)+P\left(3 / 2^{n}\right)-2 P\left(4 / 2^{n}\right)=\lambda \mu^{n-2}(\mu-\lambda) \geqq 0 .
$$

Also, $P\left(1-5 / 2^{n}\right)=1-\lambda^{n-2}(1+\lambda \mu), P\left(1-3 / 2^{n}\right)=1-\lambda^{n-1}(1+\mu), P\left(1-2^{-n}\right)=1-\lambda^{n}$, and

$$
P\left(1-5 / 2^{n}\right)+P\left(1-3 / 2^{n}\right)-2 P\left(1-4 / 2^{n}\right)=\mu \lambda^{n-2}(\mu-\lambda) \geqq 0 .
$$


Then, by Lemma 2 and (8),

Similarly,

$$
\begin{gathered}
\int_{3 / 2^{n}}^{5 / 2^{n}}(h(x)-P(x)) d x \leqq\left(\lambda / 2^{n}\right)\left((\varrho+3) P\left(4 / 2^{n}\right)+(\varrho-1) P\left(5 / 2^{n}\right)\right. \\
\left.-\varrho P\left(5 / 2^{n}\right)-(\varrho+1) P\left(4 / 2^{n}\right)-P\left(3 / 2^{n}\right)\right)=-\lambda^{2} \mu^{n-2}(\mu-\lambda) / 2^{n}
\end{gathered}
$$

$$
\int_{1-5 / 2^{n}}^{1-3 / 2^{n}}(h(x)-P(x)) d x \leqq-\mu \lambda^{n-1}(\mu-\lambda) / 2^{n},
$$

and consequently

$$
\begin{gathered}
\int_{0}^{1} h(x) d x \leqq \\
=\mu-\sum_{n=3}^{\infty} \lambda^{2} \mu^{n-2}(\mu-\lambda) 2^{-n}-\sum_{n=4}^{\infty} \mu \lambda^{n-1}(\mu-\lambda) 2^{-n} \\
=\mu-(1 / 8)\left(\mu \lambda^{2}(\mu-\lambda)(4-\mu \lambda) /(2+\mu \lambda)\right) .
\end{gathered}
$$

Since $\mu \lambda<1 / 4,(4-\mu \lambda) /(2+\mu \lambda)$ is between $5 / 3$ and 2 , the right-hand side of (13) follows. A replacement of $h(x)$ by $1-h(1-x)$ again produces the left-hand side.

\section{Upper bound of the maximal dilatation}

Linear transformations have no effect on the coefficient of quasisymmetry of $h$ or the maximal dilatation of $f_{h, r}$. There is no loss of generality in assuming that the domain of $h$ contains $[-1,1], h(0)=0, h(1)=1$, and in considering the dilatation quotient $D$ of $f_{h, r}$ at $i$. Setting $F=D+1 / D, \quad \xi=\alpha_{y}(i) / \alpha_{x}(i), \quad \eta=-\beta_{y}(i) / \beta_{x}(i), \quad \zeta=$ $\alpha_{x}(i) / \beta_{x}(i)$ one computes

where

$$
F=a(\xi, \eta, \zeta) r+b(\xi, \eta, \zeta) / r
$$

$$
\begin{aligned}
& a(\xi, \eta, \zeta)=\left((\zeta-1)^{2}+(\zeta \xi+\eta)^{2}\right) /(2 \zeta(\xi+\eta)), \\
& b(\xi, \eta, \zeta)=\left((\zeta+1)^{2}+(\zeta \xi-\eta)^{2}\right) /(2 \zeta(\xi+\eta)) .
\end{aligned}
$$

From (2) one obtains

$$
\zeta=-1 / h(-1), \quad \xi=1-\int_{0}^{1} h(x) d x, \quad \eta=1+\zeta \int_{-1}^{0} h(x) d x .
$$

We try to determine $r$ in such a way that the maximum of $F$ as $(\xi, \eta, \zeta)$ ranges over all combinations allowed by (1) and (14) is as small as possible. To this end, we first observe that $1 / \varrho \leqq \zeta \leqq \varrho$, and that a possible replacement of $h(x)$ by $h(-x) / h(-1)$ allows us to choose $\zeta \geqq 1$. By Lemma $4, \xi$ and $\eta$ both lie in $[p, q]$. The inequalities

and

$$
h(x)-h((x-1) / 2) \leqq \varrho(h((x-1) / 2)-h(-1))
$$

$$
h(x) \leqq-\varrho h(-x)
$$

follow from (1); integration over $[0,1]$ yields

$$
\zeta \leqq((2 \varrho+1) \eta-1) /(1-\xi)
$$


and

$$
\zeta \leqq \varrho(1-\eta) /(1-\xi) .
$$

Finally, the application of Lemma 4 to $(h(2 x-1)-h(-1)) /(1-h(-1))$ yields

$$
\zeta \leqq(\eta+q-p) /(\xi-q+p)
$$

for $\xi>q-p$. We denote by $T_{\varrho} \subset R^{3}$ the closed set bounded by the six planes $\zeta=1$, $\zeta=\varrho, \xi=p, \xi=q, \eta=p, \eta=q$ and the three hyperbolic paraboloids defined by equalities in (15), (16) and (17).

The expressions $a$ and $b$ can both be written in the form

$$
\left(1+\xi^{2}\right) \zeta+\left(1+\eta^{2}\right) / \zeta+g(\xi, \eta)
$$

From this it follows that for fixed $\xi, \eta, r$ the maximum of $F$ is attained at the largest possible $\zeta$ if $\eta \leqq \xi$ while for $\xi \leqq \eta$ the maximum is attained either at $\zeta=1$ or at the largest possible $\zeta$. In view of (16), the maximum is at $\zeta=1$ only if

$$
\left(1+\eta^{2}\right) /\left(1+\xi^{2}\right) \geqq \varrho(1-\eta) /(1-\xi) .
$$

One easily verifies that if $(\xi, \eta, \zeta) \subset T_{\varrho}$ and $\eta<(\varrho+1) /(3 \varrho+1)$, then (18) is not fulfilled.

If $\zeta$ is constant and $(\xi, \eta)$ is on a fixed line, $F$ can be written in the form

$$
A L+B / L+C,
$$

where $L$ is a linear expression in $\xi$ or $\eta, A>0$ and $L>0$. It follows that on any line segment in $T$ parallel to the $\xi \eta$-plane $F$ is maximized at one of the end-points. This means that for any $r$, the maximum of $F$ in $T_{\varrho}$ is attained either in one of the vertices $v_{1}=(p, p, 1), \quad v_{2}=(q-p+q / \varrho, p, \varrho), \quad v_{3}=(q, p, q / p), \quad v_{4}=(q,(1+\varrho) p-q, \varrho), \quad v_{5}=$ $(q, q, \varrho), v_{6}=(p, q, 1), v_{7}=\left(p,(\varrho+1) /(3 \varrho+1), 2 \varrho^{2} /(q(3 \varrho+1))\right), v_{8}=((\varrho+1) /(3 \varrho+1)$, $(\varrho+1) /(3 \varrho+1), \varrho)$, or on the edges $e_{i}, i=1,2,3$, joining $v_{i}$ and $v_{i+1}, e_{j}, j=4,5,6$ joining $v_{j+1}$ and $v_{j+2}$, or on $e_{7}$ which joins $v_{7}$ and $v_{1}$. Of these, $e_{1}, e_{2}, e_{4}$ and $e_{6}$ are arcs of a hyperbola while the others are linear.

On the curvilinear edges, $\eta$ is constant, and an elimination of $\xi$ makes $F$ a function of $\zeta$ with expression

$$
A(\zeta+c)+B /(\zeta+c)+C .
$$

Thus $F$ is maximized at one of the end-points. On $e_{3}, e_{5}$ and $e_{7} \xi$ is constant; elimination of $\zeta$ makes $F$ a function with expression

$$
\left(A \eta^{2}+B \eta+C\right) /\left(\eta^{2}+B_{1} \eta+C_{1}\right)
$$

lengthy but elementary computations show that $F$ is either monotonic or has at most a minimum on $e_{3}, e_{5}$ and $e_{7}$. Altogether, then, for $r$ and $\varrho$ fixed, the maximum of $F$ in $T_{\ell}$ is attained at one of the eight vertices $v_{i}$. 
Now, set

$$
\begin{gathered}
F_{i}(r)=a\left(v_{i}\right) r+b\left(v_{i}\right) / r, \quad i=1,2, \ldots, 8, \\
r_{i}=\left(b\left(v_{i}\right) / a\left(v_{i}\right)\right)^{1 / 2}, \\
r_{i j}=\left(\left(b\left(v_{i}\right)-b\left(v_{j}\right)\right) /\left(a\left(v_{j}\right)-a\left(v_{i}\right)\right)\right)^{1 / 2}, \quad i \neq j .
\end{gathered}
$$

An inspection of the graphs of $F_{i}(r)$ shows immediately the truth of

Lemma 5. Assume $b\left(v_{i}\right)>b\left(v_{j}\right)$. Then $\max _{i, j}\left\{F_{i}(r), F_{j}(r)\right\}$ is minimized at $r_{i}$ if either $r_{i j}$ is not real or $r_{i} \leqq r_{i j}$, at $r_{i j}$ if $r_{j}<r_{i j}<r_{i}$, and at $r_{j}$ if $r_{i j}<r_{j}$.

Applying Lemma 5 repeatedly one sees by routine if labourious computations that for $i=1,3,5,6,7,8$ and $\varrho>1$ the minimum of $\max \left\{F_{i}(r), F_{2}(r)\right\}$ equals $F_{2}\left(r_{2}\right)$ while for $\varrho \geqq \varrho_{0}=1.01765 \ldots$ the minimum of $\max \left\{F_{4}(r), F_{2}(r)\right\}$ also equals $F_{2}\left(r_{2}\right)$ but for $\varrho<\varrho_{0}$ the minimum equals $F_{2}\left(r_{24}\right)$. Thus we have reached upper bounds for $K\left(f_{h, r}\right)$ expressible in the form

where

$$
\begin{gathered}
D+1 / D \leqq 2\left(a\left(v_{2}\right) b\left(v_{2}\right)\right)^{1 / 2}, \quad r=r_{2}, \quad \varrho \geqq \varrho_{0}, \\
D+1 / D \leqq a\left(v_{2}\right)\left(\left(b\left(v_{2}\right)-b\left(v_{4}\right)\right) /\left(a\left(v_{4}\right)-a\left(v_{2}\right)\right)\right)^{1 / 2} \\
+b\left(v_{2}\right)\left(\left(a\left(v_{4}\right)-a\left(v_{2}\right)\right) /\left(b\left(v_{2}\right)-b\left(v_{4}\right)\right)\right)^{1 / 2}, \\
r=r_{24}, \quad 1<\varrho<\varrho_{0},
\end{gathered}
$$

$$
\begin{aligned}
& a\left(v_{2}\right)=\left((\varrho-1)^{2}+(\varrho(q-p)+1)^{2}\right) /(2(\varrho+1) q), \\
& b\left(v_{2}\right)=(\varrho+1)\left(1+(q-p)^{2}\right) /(2 q), \\
& a\left(v_{4}\right)=\left((\varrho-1)^{2}+(\varrho+p-q)^{2}\right) /(2 \varrho(\varrho+1) p), \\
& b\left(v_{4}\right)=\left(1+(q-p)^{2}\right) /(2 p q) .
\end{aligned}
$$

A routine computation shows that the inequalities

and

$$
D+1 / D \leqq 2 \varrho-1+1 /(2 \varrho-1)
$$

$$
D+1 / D \leqq \varrho^{3 / 2}+1 / \varrho^{3 / 2}
$$

for $\varrho>1$ follow from (19) and (20). We have thus proved

Theorem 1. For every @-quasisymmetric $h$ there exists a Beurling-Ahlfors extension $f_{h, r}$ with $K\left(f_{h, r}\right) \leqq \min \left\{\varrho^{3 / 2}, 2 \varrho-1\right\}$.

Remarks. 1. Numerical evidence strongly suggests that the exponent $3 / 2$ above could be lowered at least to 1.48 . The computations required for the verification seem to be exceedingly complicated.

2. If $h$ is assumed to be $\varrho$-quasisymmetric on the entire real line, additional consequencies of (1) can be derived to improve further the bounds in (13) and thus to lower the upper bound of the maximal dilatation. 


\section{A lower estimate}

For every choice of $r$, the maximal dilatation of $f_{h, r}$ is known to be at least $\varrho(h)$ [4]. Li Zhong [5] has shown that if $h_{n}(x)=x^{n}, x \geqq 0, h_{n}(x)=-|x|^{n}, x \leqq 0$, then

$$
\lim _{n \rightarrow \infty} K\left(f_{h_{n}, r}\right) / \varrho\left(h_{n}\right)=2^{2 / 3}=1.5874 \ldots .
$$

He has also given concrete examples of piecewise linear functions $h$, with $\varrho(h)>41$, such that $K\left(f_{h, r}\right)>1.4 \varrho(h)$ for every $r$. We shall exhibit piecewise linear functions $h$ such that $K\left(f_{h, r}\right)$ is always at least $1.5 \varrho(h)$ as soon as $\varrho(h)>12$.

Given $\varrho$, set $h(x)=x, x \geqq 1, h(x)=x / \varrho, x \leqq-1, h(1 / 2)=-h(-1 / 2)=\lambda$, and let $h$ be linear on $[-1,-1 / 2],[-1 / 2,1 / 2],[1 / 2,1]$. A computation shows that $\varrho(h)=\varrho$. For this $h$,

$$
\xi=(1+\lambda) / 4, \quad \eta=1-\xi, \quad \zeta=\varrho,
$$

and the dilatation quotient $D$ of $f_{h, r}$ at $i$ satisfies

$$
D+D^{-1} \geqq 2(a(\xi, \eta, \varrho) b(\xi, \eta, \varrho))^{1 / 2} .
$$

Making the substitutions (21) in (22) one arrives at

$$
(D+1 / D)^{2} \geqq(3 \varrho / 2+2 /(3 \varrho))^{2}
$$

for $\varrho>12$. The asymptotic formula

$$
\lim _{\varrho \rightarrow \infty} D / \varrho=1+(3 / 4)^{2}=1.5625
$$

is also true.

\section{References}

[1] Beurling, A., and L. V. Ahlfors: The boundary correspondence under quasiconformal mappings. - Acta Math. 96, 1956, 125-142.

[2] Goldberg, K.: A new definition for quasisymmetric functions. - Michigan Math. J. 21, 1974, $49-62$.

[3] Lehtinen, M.: A real-analytic quasiconformal extension of a quasisymmetric function. - Ann. Acad. Sci. Fenn. Ser. A. I. Math. 3, 1977, 207-213.

[4] Lehtinen, M.: The dilatation of Beurling-Ahlfors extensions of quasisymmetric functions. Ann. Acad. Sci. Fenn. Ser. A. I. Math. 8, 1983, 187-192.

[5] Li Zhong: On the Beurling-Ahlfors extension. - Acta Math. Sinica 26, 1983, 279-290 (Chinese).

[6] ReED, T.: Quasiconformal mappings with given boundary values. - Duke Math. J. 33, 1966, $459-464$.

[7] SALEM, R.: On some singular monotonic functions which are strictly increasing. - Trans. Amer. Math. Soc. 53, 1943, 427-439.

University of Helsinki

Department of Mathematics

SF-00100 Helsinki 10

Finland

Received 18 January 1984 